\title{
Identification of an autophagy-related gene signature that can improve prognosis of hepatocellular carcinoma patients
}

\author{
Xingxing Huo ${ }^{1,2,3,4}$, Jian $\mathrm{Qi}^{1,2,3}$, Kaiquan Huang ${ }^{4}$, Su Bu${ }^{4}$, Wei Yao ${ }^{4}$, Ying Chen ${ }^{4}$ and Jinfu Nie ${ }^{1,2,3^{*}}$
}

\begin{abstract}
Background: Autophagy is a programmed cell degradation mechanism that has been associated with several physiological and pathophysiological processes, including malignancy. Improper induction of autophagy has been proposed to play a pivotal role in the progression of hepatocellular carcinoma (HCC).

Methods: Univariate Cox regression analysis of overall survival (OS) was performed to identify risk-associated autophagy-related genes (ARGs) in HCC data set from The Cancer Genome Atlas (TCGA). Multivariate cox regression was then performed to develop a risk prediction model for the prognosis of 370 HCC patients. The multi-target receiver operating characteristic (ROC) curve was used to determine the model's accuracy. Besides, the relationship between drug sensitivity and ARGs expression was also examined.

Results: A total of 62 differentially expressed ARGs were identified in HCC patients. Univariate and multivariate regression identified five risk-associated ARGS (HDAC1, RHEB, ATIC, SPNS1 and SQSTM1) that were correlated with OS in HCC patients. Of importance, the risk-associated ARGs were independent risk factors in the multivariate risk model including clinical parameters such as malignant stage $(H R=1.433,95 \% \mathrm{Cl}=1.293-1.589, P<0.001)$. In addition, the area under curve for the prognostic risk model was 0.747 , which indicates the high accuracy of the model in prediction of HCC outcomes. Interestingly, the risk-associated ARGs were also correlated with drug sensitivity in HCC cell lines.
\end{abstract}

Conclusion: We developed a novel prognostic risk model by integrating the molecular signature and clinical parameters of HCC, which can effectively predict the outcomes of HCC patients.

Keywords: Autophagy, HCC, Autophagy-related genes, Molecular signature, Drug sensitivity

\section{Background}

Hepatocellular carcinoma (HCC), which accounts for $75-85 \%$ of liver cancer cases, is considered the sixth most common malignancy and the fourth with cancerrelated death worldwide [1]. The main causes of liver cancer are chronic infection with hepatitis $\mathrm{B} / \mathrm{C}$ virus, exposure to aflatoxin, alcohol abuse, and obesity [2]. HCC

\footnotetext{
* Correspondence: jeffnie@cmpt.ac.cn

${ }^{1}$ University of Science and Technology of China, Hefei, China

${ }^{2}$ Hefei Cancer Hospital, Chinese Academy of Sciences, Hefei, China

Full list of author information is available at the end of the article
}

is usually associated with poor outcomes because the treatment of HCC could be effective only when diagnosed at early stages [3]. The prognosis of HCC is currently dependent on histopathological parameters and the tumor staging system. However, such traditional approaches might not be adequate for the accurate prediction of clinical outcomes in HCC patients. Therefore, it is imperative to identify more robust and accurate prognostic indicators that can help clinicians optimize therapeutic strategies. 
Autophagy is a natural regulatory mechanism by which cells remove nonessential and dysfunctional components. It is a dynamic process that includes the induction of autophagosomes, their nucleation, double membrane growth and closure, and finally, fusion with the lysosome, which leads to disintegration of the engulfed materials [4]. Abnormal autophagy has been associated with the pathogenesis of a variety of diseases, including malignant tumors [5]. In tumors, autophagy can exert opposite environment-dependent effects, which can lead to either suppression or promotion of tumor growth [6]. Indeed, while autophagy is considered an essential gatekeeper for restricting early tumorigenesis in multiple tissues [7], defective autophagy has been shown to promote tumor proliferation in several tissues [8]. In fact, deficiency in autophagy could lead to the release of arginase I from the liver, which leads to the degradation of circulating arginine. Hence, autophagy might maintain cancer growth through circulating arginine [9].

Recent studies have reported that autophagy plays a crucial role in the pathogenesis of HCC. Indeed, autophagy levels are noticeably higher in HCC tumor tissues, compared with adjacent normal tissues. In addition, the invasion of peripheral areas by HCC tumors has been associated with higher levels of autophagy in HCC cancer cells [10]. Autophagy promotes HCC cell proliferation through the induction intracellular ATP via mitochondrial oxidative phosphorylation [11]. Despite that several indexes have been proposed for HCC prognosis [12-14], little studies have considered autophagy-related genes (ARGs) for the prediction of clinical outcomes in HCC patients. Lin et al. reported that an expression signature for ARGs related to survival prediction for HCC patients [15]. Due to individual differences in HCC patients and the expression levels of relevant genes, additional predictors of HCC prognosis are needed that are not influenced by other clinical characteristics.

\section{Methods}

\section{Patients information}

RNA-seq data and clinical information of HCC patients were obtained from The Cancer Genome Atlas (TCGA) database and The International Cancer Genome Consortium (ICGC) dataset. Genes associated with autophagy were extracted from the Human Autophagy Database (HADb), an autophagy-dedicated database that provides information on human genes involved in autophagy.

Functional annotation of differentially expressed ARGs The $\mathrm{R}$ package EdgeR was used to perform differential gene expression analysis on ARGs in the TCGA data.
ARGs exhibiting a $\log 2$ fold-change $>1$ in $\mathrm{HCC}$, compared with non-tumor tissues, and an adjusted $P<0.05$ were considered to be significantly altered. Gene ontology (GO) and Kyoto Encyclopedia of Genes and Genomes (KEGG) enrichment analysis was performed using DAVID web-tool (The Database for Annotation, Visualization and Integrated Discovery) to unveil biological attributes and signaling pathways associated with the differentially expressed ARGs. The GOplot and ClusterProfiler $\mathrm{R}$ packages were used for visualization of the selected enriched ontologies and pathways.

\section{Construction of the prognostic risk model}

Univariate cox regression analysis was used to identify differentially expressed ARGs associated with overall survival (OS) in HCC patients from the TCGA-LIHC data set. The identified OS-related ARGs were then included in a multivariate cox regression analysis to identify potential independent prognostic ARGs in HCC patients. The obtained prognostic ARGs were used to construct a risk score model. The regression coefficients in the linear formula were used as relative weights of ARG genes in the multivariate model. A risk score was calculated for each patient, a median value was identified for all patients, and HCC patients were then divided into low risk (score below the median) and high risk (score above the median) groups. The high and low risk groups were stratified and visualized using Kaplan-Meier (K-M) survival curves and analyzed for statistical significance using the log-rank test. The ARG-based risk score was finally included in a multivariate cox regression of OS to identify its prognostic value in HCC patients.

\section{Evaluation of the prognostic capacity of the model}

The survivalROC package was used to analyze the prognostic value of the ARG-based risk model in R environment. The Receiver Operating Characteristic (ROC) curve was used to check the prognostic efficiency of the risk model in survival prediction. An area under the ROC curve (AUC) was used to measure the prognostic efficiency of the model.

\section{Statistical analysis}

Data management and statistical analysis were performed using the $\mathrm{R}$ software. Plots were created using the $\mathrm{R}$ software and GraphPad Prism v7. K-M curves were plotted, and a log-rank test was applied to check for statistical differences between survival curves. A $P<$ 0.05 was used as a threshold for statistical significance.

\section{Results}

Differentially expressed ARGs

A total of 232 ARGs were identified using the HADb. A total of 370 patients with primary $\mathrm{HCC}$ had their clinical 
data and gene expression profiles available on the TCGA database (Table 1). Differential gene expression analysis identified 62 ARGs, including 58 upregulated and 4 down-regulated ARGs (Fig. 1a-b). Figure 1c shows the expression profiles of the differentially expressed ARGs in HCC and non-tumor tissue samples.

\section{Functional enrichment analysis}

Enrichment analysis was used to identify functional GO terms and KEGG pathways associated with the 62 differentially expressed ARGs in HCC samples. The GO biological processes associated with these genes were "process utilizing autophagic mechanism", and "macroautophagy", while the GO molecular functions associated with these genes were "protein kinase regulator activity", "cysteine-type endopeptidase activity", and "heat shock protein binding". Regarding cellular components, the top two enriched GO terms

Table 1 Clinical data of 370 HCC patients

\begin{tabular}{|c|c|c|c|}
\hline Clinical parameters & Variable & Total (370) & Percentages (\%) \\
\hline \multirow[t]{2}{*}{ Age } & $<65$ & 221 & $59.73 \%$ \\
\hline & $\geq 65$ & 149 & $40.27 \%$ \\
\hline \multirow[t]{2}{*}{ Gender } & Female & 121 & $32.70 \%$ \\
\hline & Male & 249 & $67.30 \%$ \\
\hline \multirow[t]{5}{*}{ Histological grade } & G1 & 55 & $14.86 \%$ \\
\hline & G2 & 177 & $47.84 \%$ \\
\hline & G3 & 120 & $32.43 \%$ \\
\hline & G4 & 13 & $3.51 \%$ \\
\hline & unknow & 5 & $1.35 \%$ \\
\hline \multirow[t]{5}{*}{ Pathological stage } & Stage I & 172 & $46.49 \%$ \\
\hline & Stage ॥ & 84 & $22.70 \%$ \\
\hline & Stage III & 85 & $22.97 \%$ \\
\hline & Stage IV & 5 & $1.35 \%$ \\
\hline & unknow & 24 & $6.49 \%$ \\
\hline \multicolumn{4}{|l|}{ TMN } \\
\hline \multirow[t]{5}{*}{ T staging } & $\mathrm{T} 1$ & 182 & $49.19 \%$ \\
\hline & $\mathrm{T} 2$ & 92 & $24.86 \%$ \\
\hline & $\mathrm{T} 3$ & 80 & $21.62 \%$ \\
\hline & $\mathrm{T} 4$ & 13 & $3.51 \%$ \\
\hline & TX/unknow & 3 & $0.81 \%$ \\
\hline \multirow[t]{3}{*}{ N staging } & NO & 252 & $68.11 \%$ \\
\hline & N1 & 4 & $1.08 \%$ \\
\hline & NX/unknow & 114 & $30.81 \%$ \\
\hline \multirow[t]{3}{*}{ M staging } & Mo & 266 & $71.89 \%$ \\
\hline & M1 & 4 & $1.08 \%$ \\
\hline & $M X$ & 100 & $27.03 \%$ \\
\hline \multirow[t]{2}{*}{ Survival status } & Dead & 125 & $33.78 \%$ \\
\hline & Alive & 245 & $67.40 \%$ \\
\hline
\end{tabular}

were "region" and "chaperone complex" (Fig. 2a). On the other hand, enrichment analysis on showed that the differentially expressed ARGs were mainly associated with the following KEGG pathways: autophagy, apoptosis, platinum drug resistance, cellular senescence, p53 signaling pathway, IL-17 signaling pathway, and protein processing in endoplasmic reticulum. An enrichment $\mathrm{z}$-score $<0$ indicated that the relationship with the pathways could be reduced (Fig. 2b). The heatmaps in Fig. 2c show the relationship between the differentially expressed ARGs and the enriched pathways.

\section{Identification of risk-associated ARGs}

The correlation between expression levels of the 62 differentially expressed ARGs and OS was evaluated using the TCGA HCC data set. Univariate cox regression was first used to identify potential prognostic differentially expressed ARGs in the HCC patients. The analysis showed that 34 ARGs had their expression levels correlated with OS (Fig. 3a). Multivariate cox proportional hazard regression analysis was then performed order to construct a prognostic model that can efficiently predict outcomes of HCC patients. Interestingly, only 5 prognosis-related ARGs (HDAC1, RHEB, ATIC, SPNS1 and SQSTM1) were identified as potential independent risk factors (Table 2). The K$\mathrm{M}$ analysis of OS showed that the high levels of HDAC1 and ATIC were strongly correlated with shorter OS time $(\mathrm{HR}=2.11$ and 2.04, respectively; 95\% $\mathrm{CI}=[1.48-3.02]$ and [1.41-2.95], respectively; $P<0.001$ for both; Fig. $3 \mathrm{~b}, \mathrm{c}$ ). Similarly, high levels of SPNS1 and SQSTM1 were also associated with poor outcomes ( $\mathrm{HR}=1.77$ and 1.70 , respectively; $95 \% \mathrm{CI}=[1.22-2.58]$ and $[1.20-2.40]$, respectively; $P<0.01$ for both; Fig. 3d, e). Likewise, high expression of RHEB was associated with shorter OS time of HCC patients $(\mathrm{HR}=1.53,95 \% \mathrm{CI}=[1.08-2.16], P=0.015$; Fig. 3f). The two risk-associated ARGs (ATIC 1 and SPNS) associate with DFS in patients with $\mathrm{HCC}(p=0.013$ and $p=$ 0.0018 , respectively). The other 3 risk-associated ARGs (SQSTM1, RHEB and HDAC1) are not significantly associated with DFS ( $p=0.087,0.061$ and 0.12 respectively) (Supplementary Fig. S1).

\section{Construction of a prognostic model using ARG genes}

A linear regression model for the calculation of prognostic risk scores using expression levels (expr) of 5 ARGs weighted by their cox regression coefficients. The risk score was calculated using the following linear formula: riskscore $=0.4216 \times$ exprHDAC1 $)+$ $(0.5443 \times$ exprRHEB $)+(0.6171 \times$ exprATIC $)+(1.3652 \times$ exprSPNS1 $)+(0.2082 \times \operatorname{exprSQSTM1}) . \quad$ A riskScore was calculated for each patient, and patients were then stratified for OS analysis into high and low risk groups relative to the median riskScore of all patients $(n=$ 


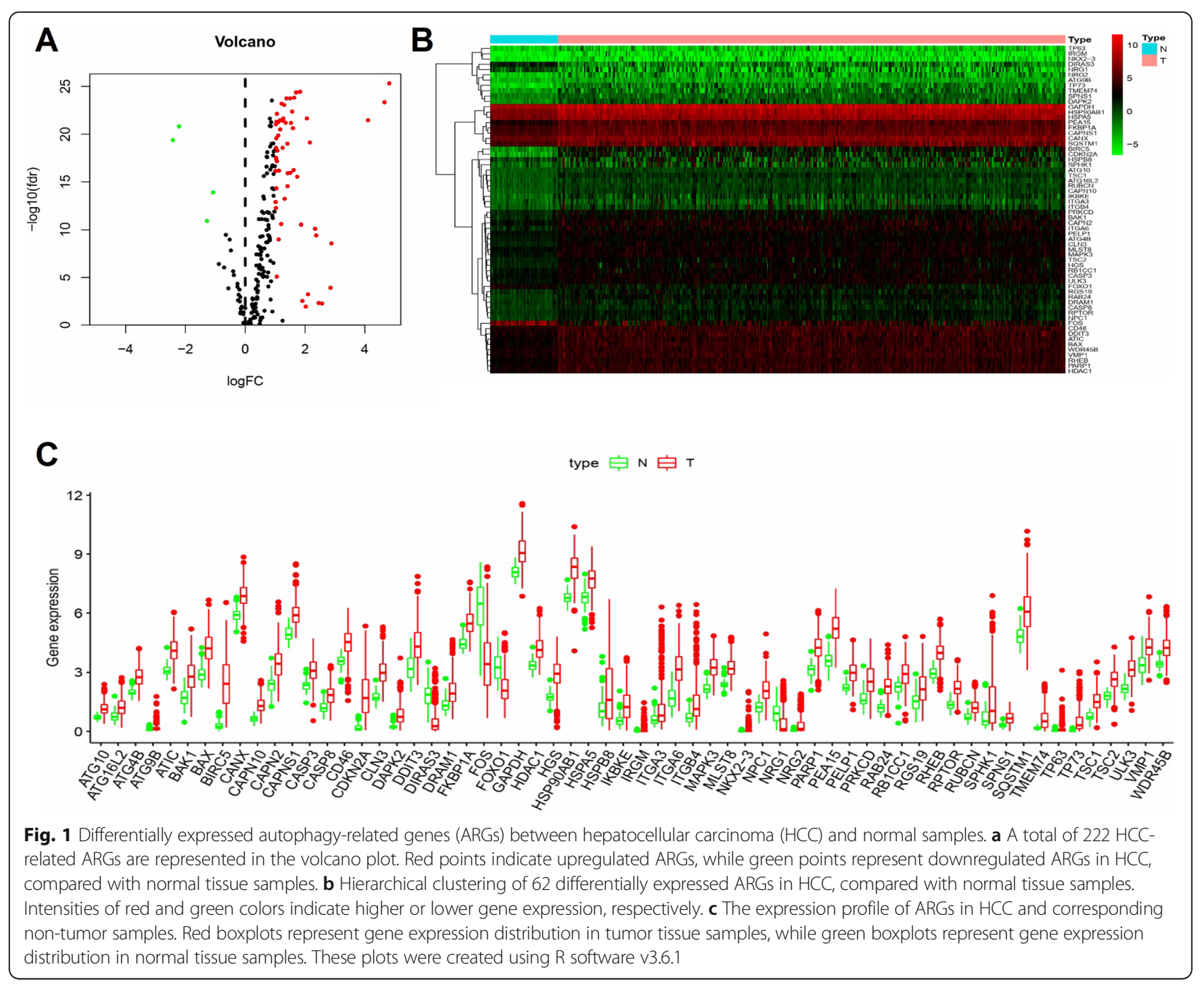

185). The K-M curve, along with the log rank test, indicated that the low-risk group exhibited favorable outcomes, while the high-risk group was associated with unfavorable outcomes $(p<0.001$; Fig. 4a). The distribution and status of OS was then analyzed by ranking the risk scores (Fig. 4b-c). Figure $4 \mathrm{~d}$ shows the expression profiles of risk-associated ARGs in high-risk and low-risk HCC patient groups.

\section{Significance of the ARG-based risk model as an independent risk factor}

The correlation of the clinical characteristics of patients and the riskScore with OS was then analyzed using univariate and multivariate regression analysis. Univariate cox regression analysis showed that the pathological stage, the $\mathrm{T}$ stage, the $\mathrm{M}$ stage and the riskScore were correlated with OS of HCC patients $(p<0.05$; Fig. 5a). Of importance, multivariate cox regression analysis including clinical parameters and riskScores showed that only the riskScore was independently associated with OS of HCC patients ( $p<0.001$; Fig. 5b).
The prognostic efficiency of the ARG-based risk model A multi-target ROC curve was performed to evaluate the prognostic efficiency of the risk model in the prediction of clinical outcomes in HCC patients. As shown in Fig. 6a, the AUC for the risk score was 0.747 , which indicates a competitive performance.

The correlation between the risk score and clinical parameters was then analyzed. The results showed that the riskScore was higher in histological stages III-IV, compared with stages I-II $(P=0.040$; Fig. 6b). In addition, the riskScore was higher in T3-T4 stages, compared with T1-T2 stages $(P=0.038$; Fig. $6 c)$. On the other hand, no differences in the riskScore were observed between patients $>65$ and those $\leq 65$ years old $(P=0.916)$, between male and female patients $(P=0.596)$, between grades $\mathrm{G} 1-\mathrm{G} 2$ and G3G4 $(P=0.119)$, between stages $\mathrm{N} 1$ and N0 $(P=$ $0.573)$, or between stages M1 and M0 $(P=0.348)$ (Fig. 6d-h). 
A
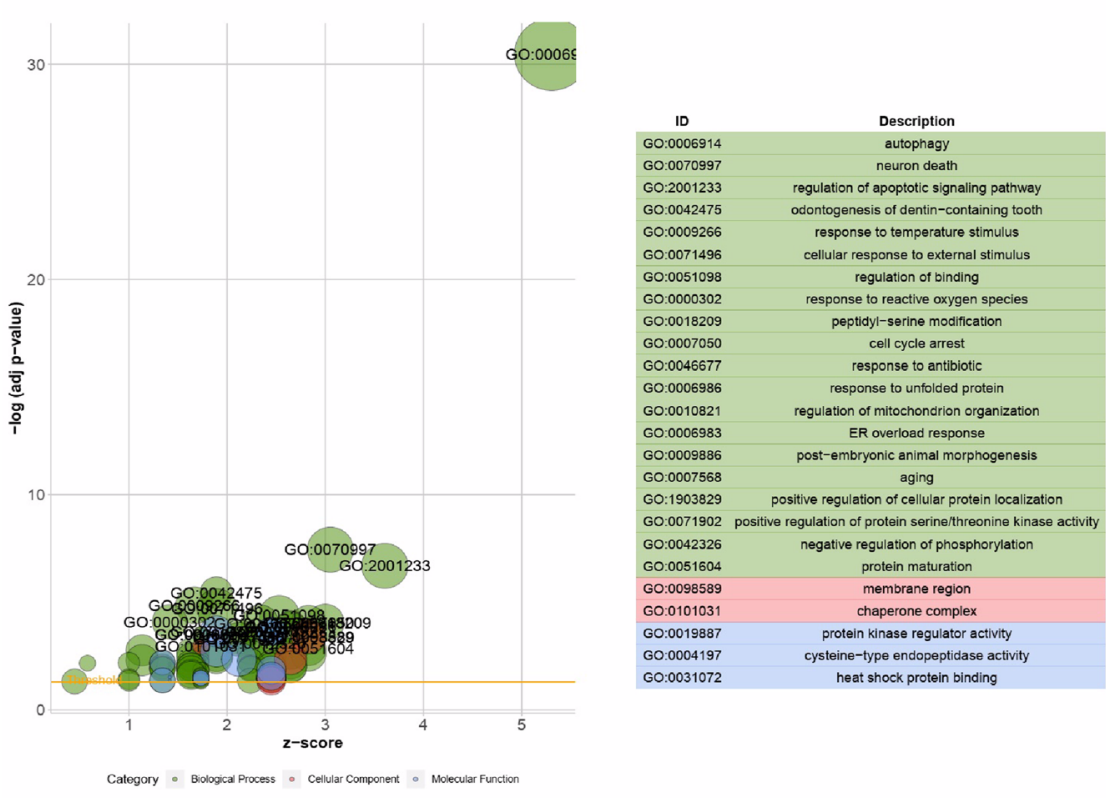

B

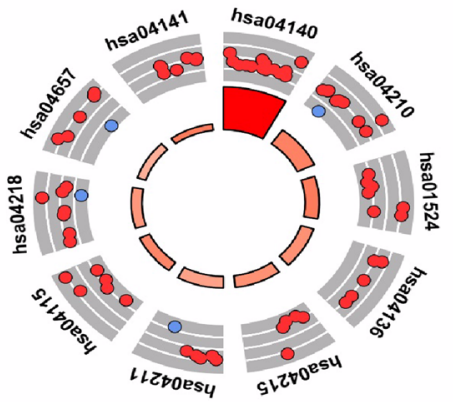

\begin{tabular}{|r|r|}
\hline \multicolumn{1}{|c|}{ ID } & $\begin{array}{c}\text { Description } \\
\text { hsa04140 }\end{array}$ \\
\hline hsa04210 & $\begin{array}{r}\text { Autophagy - animal } \\
\text { Apoptosis }\end{array}$ \\
\hline hsa01524 & $\begin{array}{r}\text { Platinum drug resistance } \\
\text { Autophagy - other }\end{array}$ \\
\hline hsa04136 & $\begin{array}{r}\text { Apoptosis - multiple species } \\
\text { hsa04215 }\end{array}$ \\
\hline hsa04211 & Longevity regulating pathway \\
\hline hsa04115 & p53 signaling pathway \\
\hline hsa04218 & Cellular senescence \\
\hline hsa04657 & IL-17 signaling pathway \\
\hline hsa04141 & Protein processing in endoplasmic reticulum \\
\hline
\end{tabular}

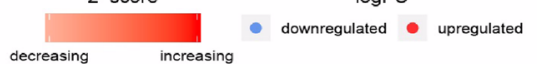

C

$$
\begin{array}{r}
\text { Toll-like receptor signaling pathway- } \\
\text { TNF signaling pathway- } \\
\text { Protein processing in endoplasmic reticulum- } \\
\text { Platinum drug resistance- } \\
\text { PI3K-Akt signaling pathway- } \\
\text { Phospholipase D signaling pathway- } \\
\text { p53 signaling pathway- } \\
\text { Necroptosis- } \\
\text { mTOR signaling pathway- } \\
\text { Longevity regulating pathway- } \\
\text { IL-17 signaling pathway- } \\
\text { Endocrine resistance- } \\
\text { Cellular senescence- } \\
\text { EGFR tyrosine kinase inhibitor resistance- } \\
\text { C-type lectin receptor signaling pathway- } \\
\text { Autophagy - other- } \\
\text { Autophagy - animal- } \\
\text { Apoptosis - multiple species- } \\
\text { Apoptosis- }
\end{array}
$$

AMPK signaling pathway-

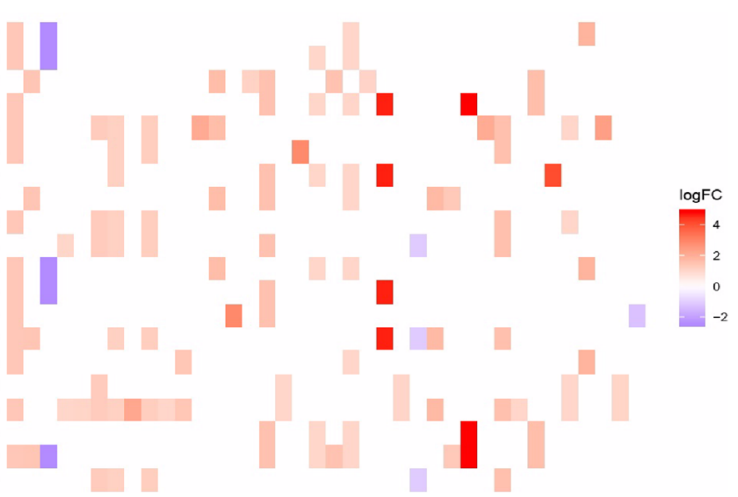

Fig. 2 Functional enrichment analysis of the differentially expressed ARGs. a The enriched Gene ontology (GO) terms are shown in a bubble plot. The size of the displayed circles is proportional to the number of genes assigned to the term. Green circles represent biological process terms, red circles represent cellular component terms, and blue circles represent molecular function terms. b The outer circle shows a scatter diagram the logFC allocated to each term. c The heatmap shows the relationship between differentially expressed ARGs and the enriched Kyoto Encyclopedia of Genes and Genomes (KEGG) pathways. The heatmap colors represent logFC value of each gene in HCC, compared with normal samples. These plots were created using R software v3.6.1 

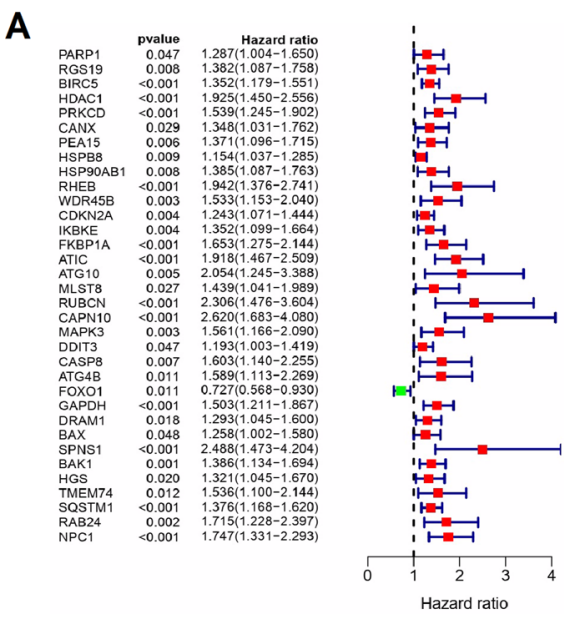

C

ATIC (471)

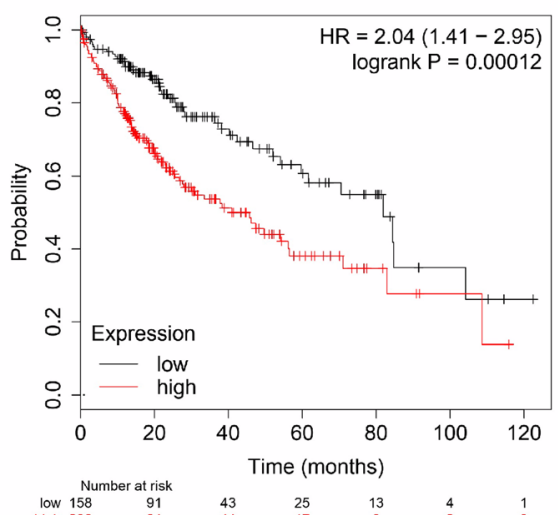

E

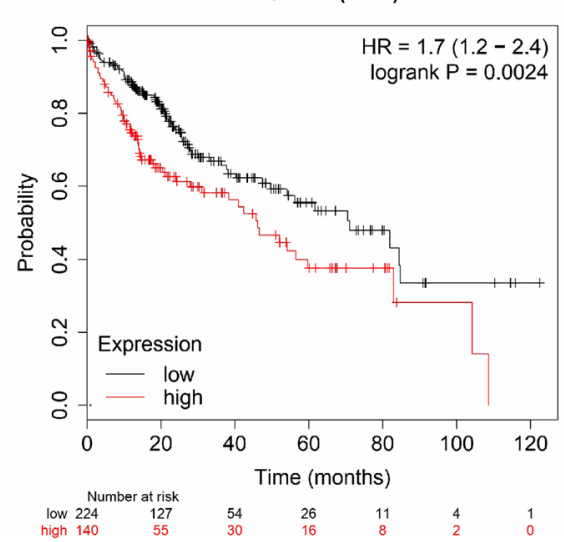

B

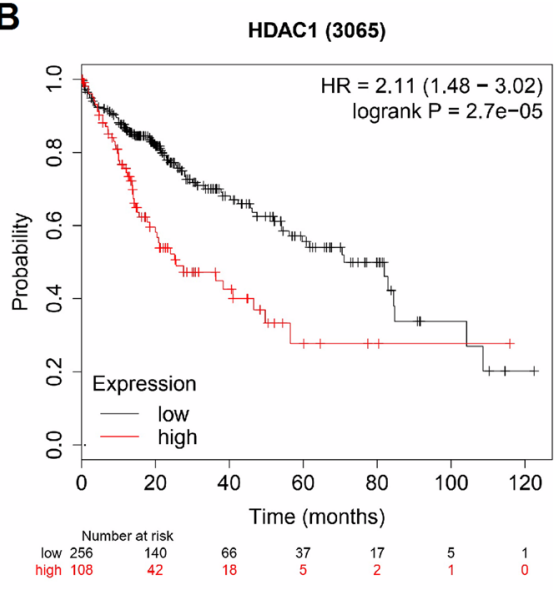

D

SPNS1 (83985)

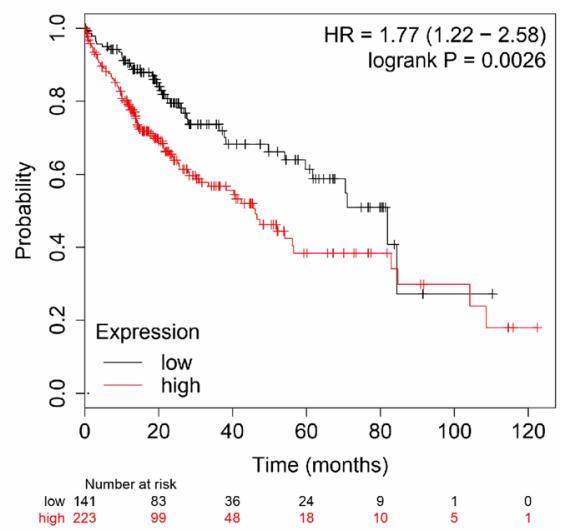

F

RHEB (6009)

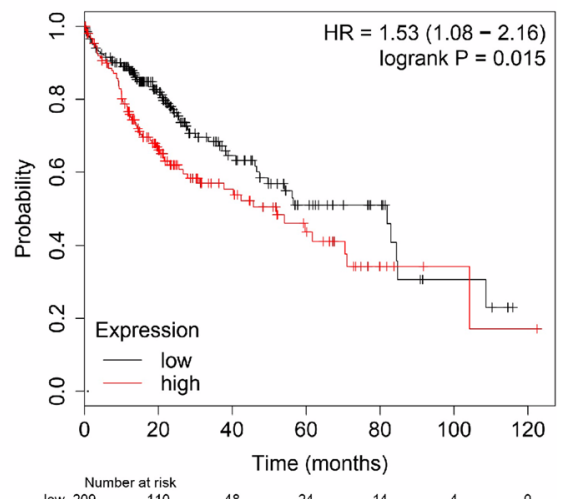

Fig. 3 Univariate cox regression and Kaplan-Meier (K-M) survival curves of the differentially expressed ARGs. a A total of 34 ARGs were correlated with overall survival $(\mathrm{OS})$ times of HCC patients $(P<0.05)$. b-f K-M curves showing the OS probability of patients stratified based on their expression of HDAC1, RHEB, ATIC, SPNS1 and SQSTM1, respectively

Verification of the ARG-based risk model in the testing group

We further evaluated prognostic efficiency of the ARGbased risk model by analyzing the patients in the different liver cancer cohorts from ICGC dataset (https://dcc.
icgc.org/releases/current/Projects/LIRI-JP). For 232 LIRI-JP samples, patients in high-risk group had inferior OS than patients in low-risk group $(p=0.0045)$ (Fig. 7a). The distribution and status of OS and expression profiles of risk-associated ARGs were also analyzed by 
Table 2 Multivariate cox regression analysis data of the prognosis-related ARGs in HCC

\begin{tabular}{llll}
\hline Gene & coef & HR $(95 \% \mathrm{Cl})$ & $\boldsymbol{P}$-value \\
\hline HDAC1 & 0.42 & $1.52(1.08-2.16)$ & 0.017281 \\
RHEB & 0.54 & $1.72(1.16-2.55)$ & 0.006484 \\
ATIC & 0.62 & $1.85(1.29-2.66)$ & $<0.001$ \\
SPNS1 & 1.37 & $3.92(1.62-9.49)$ & 0.002491 \\
SQSTM1 & 0.21 & $1.23(1.03-1.48)$ & 0.025931 \\
\hline
\end{tabular}

ranking the risk scores in high-risk and low-risk HCC patient groups from ICGC dataset (Fig. 4b-d). Overall, the accuracy of ARGs-based risk model was confirmed in the independent validation liver cancer cohorts.

\section{The relationship of the drug sensitivity and risk ARGs}

The relationship between drug sensitivity of 17 HCC cell lines and the relative expression levels of risk-associated ARGs was explored using data available from The Genomics of Drug Sensitivity of Cancer Database (GDSC). We further analyzed the correlation between the expression of HDAC1, RHEB and SQSTM1 with the IC50 of specific targeted drugs. We presumed that a positive correlation between the expression of these genes and the IC50 of the studied drugs would indicate a basis for developing drug resistance in HCC patients. In contrast, a negative correlation between risk-associated ARGs and IC50 would indicate higher drug sensitivity in HCC cell lines. High HDAC1 expression was associated with higher drug resistance (higher IC50) of HCC cell lines to Trametinib, 17-AAG, HG-5-113-01, Bleomycin, RDEA119, Nutlin-3a, PD-0325901, Elesclomol, CHIR99021, Afatinib, Cetuximab and Selumetinib $(p<0.05)$, while it was associated with higher drug sensitivity (lower IC50) of HCC cell lines to Pyrimethamine and Methotrexate ( $p<0.05$; Fig. 8a).

On the other hand, higher RHEB expression resistance to ha-793,887 in HCC cell lines $(p<0.05)$, while it was associated with higher sensitivity to 17-AAG, Elesclomol, PD-0325901, Docetaxel, Trametinib, RDEA119 and Selumetinib ( $p<0.05$; Fig. $8 \mathrm{~b})$. Furthermore, higher SQSTM1 expression was associated with higher resistance of HCC cell lines to GSK1070916 ( $p<0.05)$, while it was associated with higher sensitivity to other drugs such as 17-AAG, Trametinib, RDEA119, PD-0325901, Selumetinib, Dasatinib, Docetaxel and Lapatinib $(p<$ 0.05; Fig. 8c).

\section{Discussion}

The role of autophagy in maintaining genome integrity and cellular metabolism and homeostasis has been well demonstrated; however, its prognostic significance in human malignant tumors has not been fully explored $[16,17]$. Autophagy can maintain the survival of tumor cells under stress, and hence, promote tumor progression. Despite that endogenous tumor factors and exogenous interventions to promote or suppress autophagy have been proposed as potential cancer treatments [4], autophagy-targeting cancer therapies remain controversial. Previous studies have reported that differential translation of autophagy-related transcripts may lead to malfunctional autophagosome in HCC cells [18]. Autophagy activation can promote the proliferation of HCC cells through JNK1/Bcl-2 signaling [19]. In addition, autophagy can promote metastasis through Wnt/ $\beta$-catenin signaling [20] and via the induction of epithelial-mesenchymal transition [21]. Autophagy is considered an important mechanism of drug resistance by supporting the survival of cancer cells under metabolic and therapeutic stress [22]. In fact, sorafenib, the only drug approved for the treatment of advanced HCC, may promote autophagy in HCC cells through cellular protein networks. Luo et al. reported that the combination of PSMD10 and Atg7 could be used as a prognostic predictor in HCC patients receiving sorafenib therapy [23]. In addition, the expression level of the autophagyrelated marker LC3 has been associated with poor outcomes in HCC patients receiving surgical resection [24].

In this study, the high-throughput transcriptomics data of HCC patients were analyzed to identify potential prognostic ARGs. A total of 62 ARGs were differentially expressed in HCC patient tumor samples, compared with normal tissues, including 58 up-regulated and 4 down-regulated genes. Univariate cox regression analysis was then performed on these genes to identify 34 ARGs that were correlated with OS of HCC patients. Of these, 5 risk-associated differentially expressed ARGs (HDAC1, RHEB, ATIC, SPNS1 and SQSTM1) were further identified using multivariate cox regression analysis and were used to construct a prognostic model for the riskstratification of HCC patients based on a weighted risk score. Survival analysis showed that low-score groups exhibited better OS, compared with patients in highscore group. The multi-target ROC curve was then performed to validate the prognostic significance of the model, which was further analyzed for its correlation with clinical parameters of HCC patients. Previous work revealed that the 3 ARGs BIRC5, FOXO1 and SQSTM1 were associated with OS in HCC patients. HCC patients were stratified based on pathological stage [15]. Furthermore, results suggest that the risk score could significantly stratify HCC patients based on their histological and T-based staging systems.

HDAC1, a member of the histone deacetylase (HDACs) family, has been shown to play a crucial role in the epigenetic regulation of key oncogenes through the form a closed chromatin structure via histone deacetylation. A growing line of evidence has shown that 

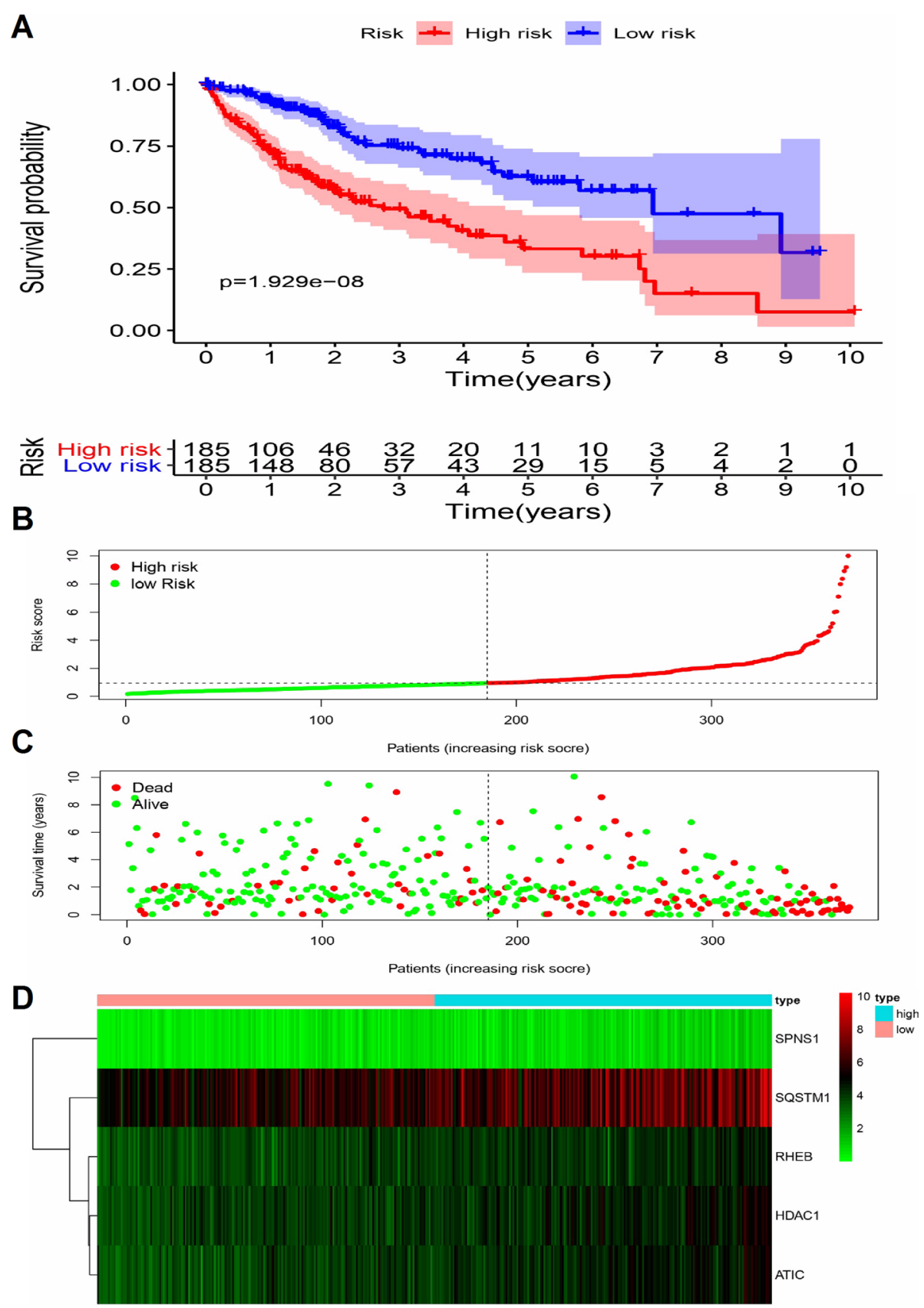

Fig. 4 Correlation between the prognostic risk model and HCC patients' survival probability. a K-M plot shows that patients in the high-score group had remarkably shorter OS time, compare with patients in the low-score group. b-c The distribution of risk score with patient's survival outcomes. d Heatmap showing the expression profiles of the five risk-associated ARGs. These plots were created using R software v3.6.1

HDAC1 could affect various oncogenic processes, such as cell proliferation and invasion, in multiple malignant tumors. The down-regulation of homeobox A10 has been shown to inhibit the proliferation of HCC cells and induce cell cycle arrest through the regulation of HDAC1 expression [25]. In addition, the transcription factor Yin-Yang 1 has been reported to reduce sensitivity of $\mathrm{HCC}$ cells to treatment by inducing HDAC1 expression [26]. Furthermore, miR-34a was demonstrated to inhibit cellular proliferation and induce apoptosis by down-regulation of HDAC1 expression in HCC cells [27]. A meta-analysis showed that high expression of HDAC1 is associated with poor OS in gastrointestinal and lung cancers, which indicates that HDAC1 may serve as a prognostic signature in these malignancies $[28,29]$. 


$\begin{array}{lrr}\text { A } & \text { pvalue } & \text { Hazard ratio } \\ \text { age } & 0.591 & 1.005(0.987-1.023) \\ \text { gender } & 0.301 & 0.780(0.487-1.249) \\ \text { grade } & 0.914 & 1.017(0.746-1.387) \\ \text { stage } & <0.001 & 1.865(1.456-2.388) \\ \mathrm{T} & <0.001 & 1.804(1.434-2.270) \\ \mathrm{M} & 0.023 & 3.850(1.207-12.281) \\ \mathrm{N} & 0.328 & 2.022(0.494-8.276) \\ \text { riskScore } & <0.001 & 1.454(1.325-1.595)\end{array}$

$\begin{array}{lrr}\text { B } & \text { pvalue } & \text { Hazard ratio } \\ \text { age } & 0.597 & 1.005(0.986-1.025) \\ \text { gender } & 0.597 & 0.867(0.512-1.469) \\ \text { grade } & 0.919 & 0.983(0.701-1.378) \\ \mathrm{T} & <0.001 & 1.602(1.244-2.063) \\ \mathrm{M} & 0.410 & 1.712(0.477-6.144) \\ \mathrm{N} & 0.307 & 2.122(0.501-8.986) \\ \text { riskScore } & <0.001 & 1.429(1.289-1.584)\end{array}$
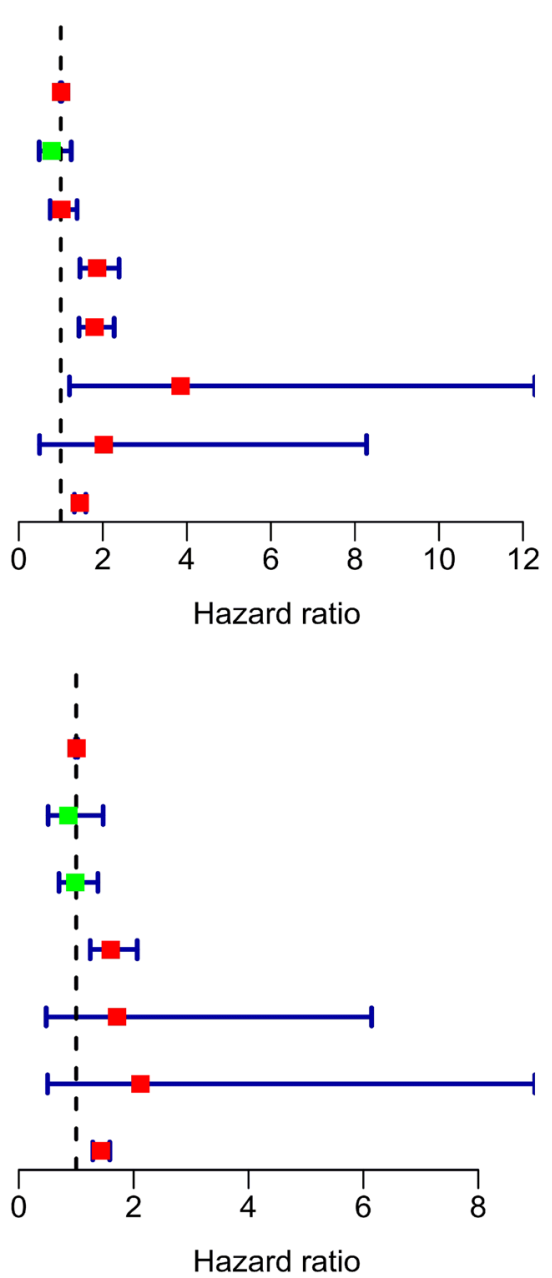

Fig. 5 Univariate and multivariate regression analysis of overall survival (OS). (a) Univariate and (b) multivariate cox regression analyses show that the prognostic risk score was independently correlated with OS $(P<0.001)$. Forest plot showing the association between risk factors and OS in HCC patients. These plots were created using R software v3.6.1

Our results showed that RHEB, a key regulator of mTOR signaling, exhibited a high expression level in cancer samples, compared with normal and adjacent normal samples. Previous analysis of cancer cytogenetic and transcriptomic databases indicated that RHEB mRNA expression was up-regulated in different carcinoma histotypes and was associated with poor outcomes in multiple types of malignancies [30]. Besides, RHEB expression was associated with higher cancer stages, higher mortality, tumor differentiation and pathological satellites in patients with hepatitis Brelated HCC [31, 32].

Previous studies have reported that ATIC is a bifunctional protease that catalyzes the last two steps in the purine biosynthesis pathway. Depletion of ATIC or suppression of its transformylase activity significantly decreased the survival rate of cells in clonogenic survival assays, which indicates that ATIC may promote the proliferation and migration in cancer cell lines [33]. Indeed, suppression of ATIC expression significantly inhibited the ability of HCC cells to proliferate and migrate through the regulation of the AMPK-mTOR-S6K1 signaling pathway. Therefore, in line with our results, the high expression of ATIC could be positively correlated with adverse prognosis in HCC patients [34].

SQSTM1 has been reported as a potential oncogene in various cancers, including HCC. p62, the gene product of SQSTM1, is a versatile protein that acts as an adaptor that induces the degradation of specific active molecules through autophagy [35]. Wei et al. reported that SQST M1 contributes to the development of autophagydeficient cancers via NF-kappaB pathway. Therefore, targeting autophagy and the autophagy-associated SQST M1 gene expression could be exploited for developing more effective cancer treatments [36]. Indeed, 

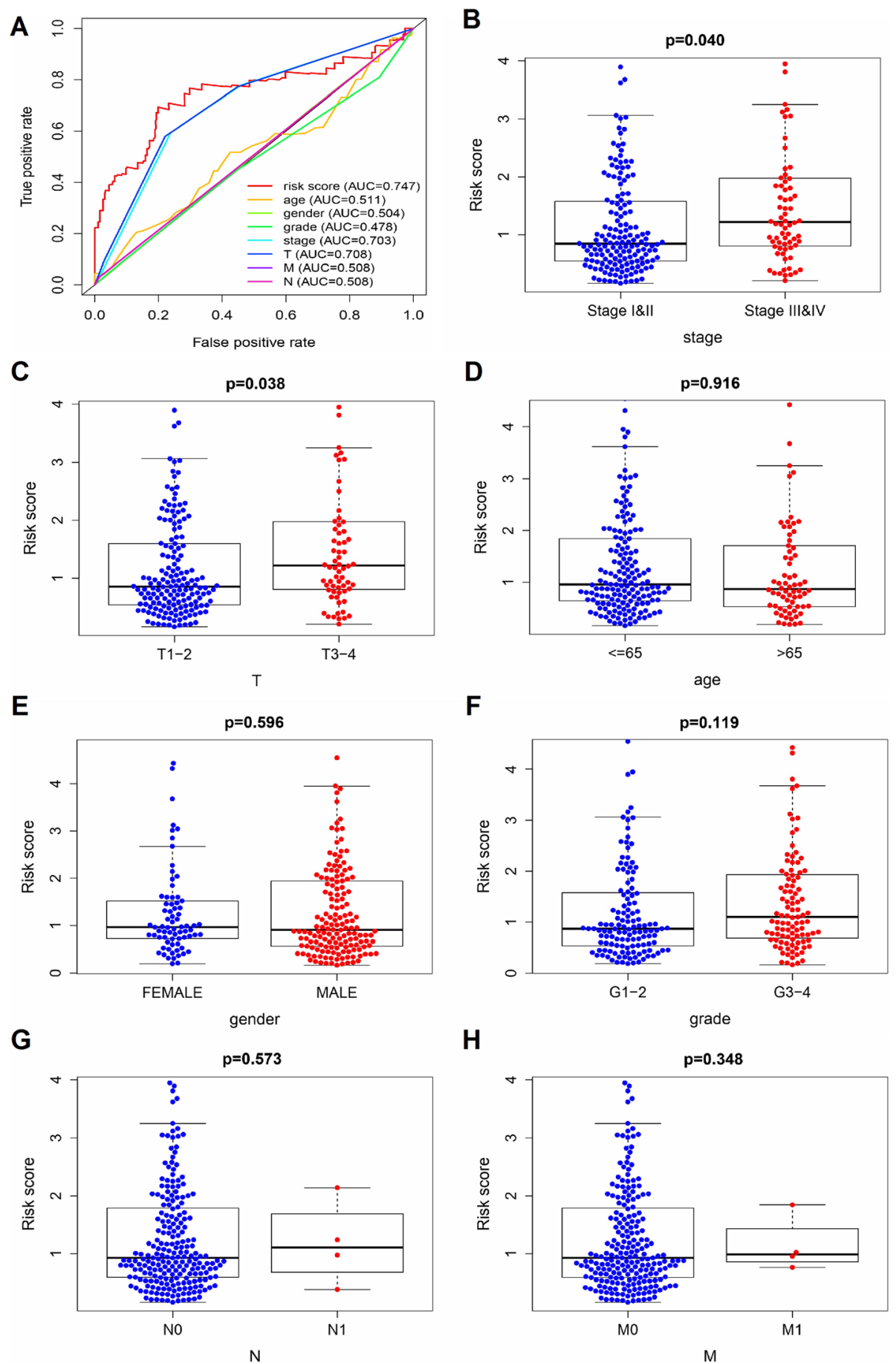

Fig. 6 The prognostic efficiency of risk-associated ARGs in HCC patients. a Multi-target receiver operating characteristic (ROC) curve of the sensitivity and specificity of OS prediction based on the prognostic risk model in HCC patients. The clinicopathological significance of the risk score in HCC patients in relation with (b) cancer stages, (c) pathological T stages, (d) age, (e) gender, (f) histological grades, (g) pathological N stages, and (h) pathological M stages. These plots were created using R software v3.6.1

phosphorylated SQSTM1/p62 has been shown to accumulate in the HCC tumor region, while its inhibitor may inhibit cell proliferation and resistance to anticancer agents [37]. Furthermore, multiple studies reported that
SQSTM1 could serve as a novel prognostic biomarker in multiple cancers types, including nasopharyngeal carcinoma, lung cancer, oral squamous cell carcinoma, and HCC [38-41]. 


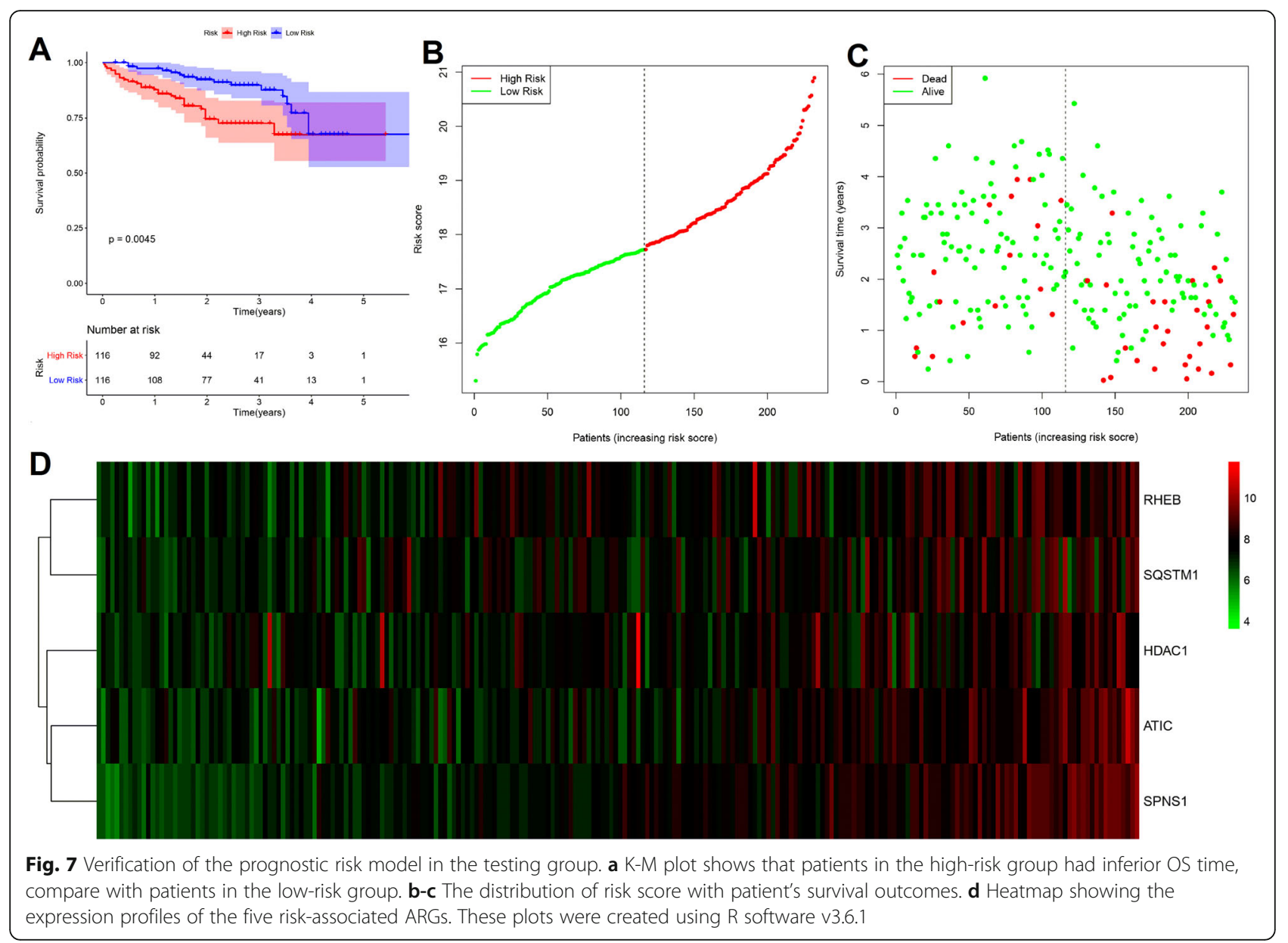

SPNS1 (Spinster homolog 1) is a hypothetical lysosomal $\mathrm{H}^{+}$-carbohydrate transporter that functions in late stage macroautophagy in vertebrates [42]. In this study, SPNS1 showed the greatest contribution to outcome predictions compared to the other 4 genes analyzed. In addition to OS, K-M analysis for DFS showed that high levels of SPNS1 also correlated with shorter DFS time $(p=0.013)$. Yanagisawa et al. reported that upregulation of SPNS1 regulates luminal solute compositions, thereby altering the subcellular distribution of lysosomes and the accumulation of p62 [43]. Dysregulation of autophagy lysosomes may promote the invasion and migration of HCC [44].

In the study presented here, we demonstrate the relationship between drug sensitivity of 17 HCC cell lines and the relative expression levels of risk-associated ARGs using the GDSC database. Even though many of these drugs are not in clinical use, identifying correlations between risk-associated ARGs and drug sensitivity may identify putative therapeutic biomarkers for further validation. Alterations in cancer genomes can influence clinical outcome to anticancer treatment. However, many cancer drugs already used and under development are not associated with specific genomic markers that can guide clinical application to maximize patient benefit [15]. In present study, we postulate that HDAC1 is a potential therapeutic target for HCC patients since high HDAC1 expression was associated with elevated drug resistance. By molecularly stratifying patient populations, drug sensitivity information can optimize the design of clinical trials and ultimate success of anticancer treatment.

\section{Conclusion}

In conclusion, we have identified 5 prognostic riskassociated ARGs (HDAC1, RHEB, ATIC, SPNS1 and SQSTM1) by correlating the molecular signature of ARG with clinical outcomes of HCC patients. The identified risk-associated ARGs could provide a basis for the development of HCC therapeutic interventions via autophagy-related mechanisms. Of importance, we constructed a novel risk model that can robustly stratify HCC patients into risk groups. Nevertheless, further prospective experiments are required to further confirm the clinical value of this model in defining the optimal personalized targeted treatment. 


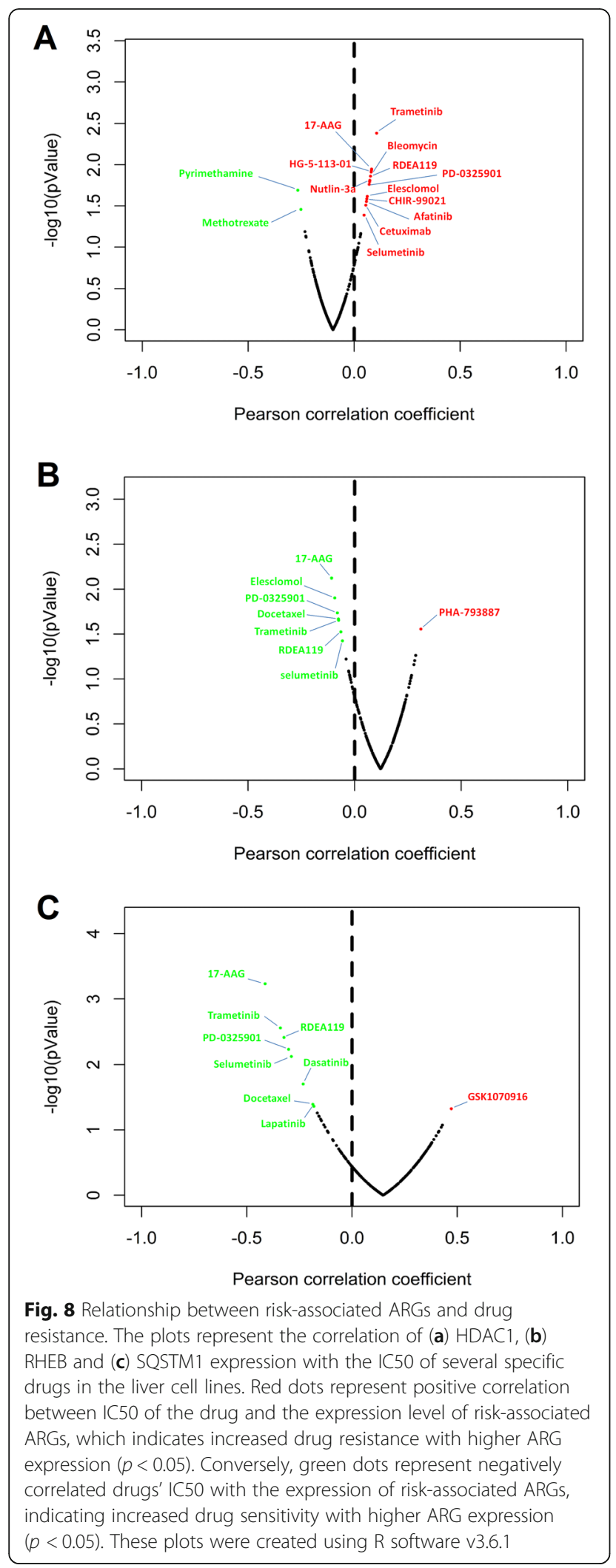

\section{Supplementary information}

Supplementary information accompanies this paper at https://doi.org/10. 1186/s12885-020-07277-3

Additional file 1 Figure S1. Disease free survival (DFS) curves of the differentially expressed ARGs in HCC patients. (A-E) K-M curves showing the DFS probability of patients stratified based on their expression of ATIC, SPNS1, SQSTM1, RHEB and HDAC1 respectively.

\section{Abbreviations}

HCC: Hepatocellular carcinoma; OS: Overall survival; DFS: Disease free survival; ARGs: Autophagy-related genes; TCGA: The Cancer Genome Atlas; ICGC: The International Cancer Genome Consortium; HADb: Human Autophagy Database; GO: Gene ontology; KEGG: Kyoto Encyclopedia of Genes and Genomes; K-M: Kaplan-Meier; ROC: Receiver Operating Characteristic; AUC: area under the ROC curve; expr: Expression levels; GDSC: Genomics of Drug Sensitivity of Cancer Database

\section{Acknowledgements}

Not applicable.

\section{Authors' contributions}

Conceived and designed the experiments: JFN. Analyzed the data: XXH, JQ YC, YW and KQH. Performed data analysis and created the figures: SB. Wrote the manuscript: XXH and JQ. The authors read and approved the final manuscript.

\section{Funding}

This study was supported by the 100-Talent Program of Chinese Academy of Sciences financed study designing and data analysis. The National Natural Science Foundation of China (No. 81803938) and Anhui University of Traditional Chinese Medicine Natural Science Foundation (No. 2018zryb48 and No. 2019zryb12) financed data collection, interpretation and writing the manuscript.

\section{Availability of data and materials}

The mRNA expression and clinical information of HCC were mined from TCGA-LIHC were available in the Genomic Data Commons Data Portal (National Cancer Institute, NIH, USA) repository. All relevant materials are provided in the manuscript.

Ethics approval and consent to participate

The data of HCC patients we used in this study are all from the publicly accessible TCGA database. The TCGA offer anonymous data.

\section{Consent for publication}

Not applicable.

\section{Competing interests}

The authors declare that they have no competing interests.

\section{Author details}

'University of Science and Technology of China, Hefei, China. ${ }^{2}$ Hefei Cancer Hospital, Chinese Academy of Sciences, Hefei, China. ${ }^{3}$ Anhui Province Key Laboratory of Medical Physics and Technology, Institute of Health and Medical Technology, Hefei Institutes of Physical Science, Chinese Academy of Sciences, Hefei, China. ${ }^{4}$ Experimental Center of Clinical Research, the First Affiliated Hospital of Anhui University of Chinese Medicine, Hefei, China.

Received: 17 February 2020 Accepted: 9 August 2020 Published online: 17 August 2020

\section{References}

1. Bray F, Ferlay J, Soerjomataram I, Siegel RL, Torre LA, Jemal A. Global cancer statistics 2018: GLOBOCAN estimates of incidence and mortality worldwide for 36 cancers in 185 countries. CA Cancer J Clin. 2018;68(6):394-424.

2. Chimed T, Sandagdorj T, Znaor A, Laversanne M, Tseveen B, Genden P, Bray $F$. Cancer incidence and cancer control in Mongolia: results from the National Cancer Registry 2008-12. Int J Cancer. 2017;140(2):302-9. 
3. Bruix J, Sherman M. American Association for the Study of liver D: management of hepatocellular carcinoma: an update. Hepatology. 2011; 53(3):1020-2.

4. Levy JMM, Towers CG, Thorburn A. Targeting autophagy in cancer. Nat Rev Cancer. 2017;17(9):528-42.

5. Amaravadi RK, Kimmelman AC, Debnath J. Targeting autophagy in Cancer: recent advances and future directions. Cancer Discov. 2019;9(9):1167-81.

6. Bryant $\mathrm{KL}$, Der CJ. Blocking autophagy to starve pancreatic cancer. Nat Rev Mol Cell Biol. 2019;20(5):265.

7. Liu H, He ZY, Simon HU. Protective role of autophagy and autophagyrelated protein 5 in early tumorigenesis. J Mol Med. 2015;93(2):159-64

8. Chen N, Eritja N, Lock R, Debnath J. Autophagy restricts proliferation driven by oncogenic phosphatidylinositol 3-kinase in three-dimensional culture. Oncogene. 2013;32(20):2543-54.

9. Poillet-Perez L, Xie X, Zhan L, Yang Y, Sharp DW, Hu ZS, Su X, Maganti A Jiang C, Lu W, et al. Autophagy maintains tumour growth through circulating arginine. Nature. 2018;563(7732):569-73.

10. Chen DP, Ning WR, Li XF, Wei Y, Lao XM, Wang JC, Wu Y, Zheng LM. Peritumoral monocytes induce cancer cell autophagy to facilitate the progression of human hepatocellular carcinoma. Autophagy. 2018;14(8):1335-46.

11. Toshima T, Shirabe $K$, Matsumoto $Y$, Yoshiya $S$, Ikegami T, Yoshizumi T, Soejima Y, Ikeda T, Maehara Y. Autophagy enhances hepatocellular carcinoma progression by activation of mitochondrial beta-oxidation. J Gastroenterol. 2014;49(5):907-16.

12. Yu J, Li X, Tao Q, Yu XL, Cheng ZG, Han ZY, Guo M, Liang P. Hypermethylation of ZNF545 is associated with poor prognosis in patients with early-stage hepatocellular carcinoma after thermal ablation. Gut. 2015; 64(11):1836-7.

13. Xiao S, Chang RM, Yang MY, Lei X, Liu X, Gao WB, Xiao JL, Yang LY. Actinlike $6 \mathrm{~A}$ predicts poor prognosis of hepatocellular carcinoma and promotes metastasis and epithelial-mesenchymal transition. Hepatology. 2016;63(4): 1256-71.

14. Sun H, Huang Q, Huang M, Wen H, Lin R, Zheng M, Qu K, Li K, Wei H, Xiao W, et al. Human CD96 correlates to natural killer cell exhaustion and predicts the prognosis of human hepatocellular carcinoma. Hepatology. 2019;70(1):168-83.

15. Yang W, Soares J, Greninger P, Edelman EJ, Lightfoot H, Forbes S, Bindal N, Beare D, Smith JA, Thompson IR, et al. Genomics of drug sensitivity in Cancer (GDSC): a resource for therapeutic biomarker discovery in cancer cells. Nucleic Acids Res. 2013;41(Database issue):D955-61.

16. Boya $P$, Reggiori F, Codogno P. Emerging regulation and functions of autophagy. Nat Cell Biol. 2013;15(7):713-20.

17. Poillet-Perez $L$, White $E$. Role of tumor and host autophagy in cancer metabolism. Genes Dev. 2019;33(11-12):610-9

18. Ji E, Kim C, Kang H, Ahn S, Jung M, Hong Y, Tak H, Lee S, Kim W, Lee EK RNA Binding Protein HuR Promotes Autophagosome Formation by Regulating Expression of Autophagy-Related Proteins 5, 12, and 16 in Human Hepatocellular Carcinoma Cells. Mol Cell Biol. 2019;39(6):e00508-18.

19. Deng G, Zeng S, Qu Y, Luo Q, Guo C, Yin L, Han Y, Li Y, Cai C, Fu Y, et al. BMP4 promotes hepatocellular carcinoma proliferation by autophagy activation through JNK1-mediated BCl-2 phosphorylation. J Exper Clin Cancer Res. 2018;37(1):156.

20. Fan Q, Yang L, Zhang X, Ma Y, Li Y, Dong L, Zong Z, Hua X, Su D, Li H, et al. Autophagy promotes metastasis and glycolysis by upregulating MCT1 expression and Wnt/beta-catenin signaling pathway activation in hepatocellular carcinoma cells. J Exper Clin Cancer Res. 2018;37(1):9.

21. Li J, Yang B, Zhou Q, Wu Y, Shang D, Guo Y, Song Z, Zheng Q, Xiong J. Autophagy promotes hepatocellular carcinoma cell invasion through activation of epithelial-mesenchymal transition. Carcinogenesis. 2013;34(6): 1343-51.

22. Thorburn A, Thamm DH, Gustafson DL. Autophagy and cancer therapy. Mol Pharmacol. 2014;85(6):830-8.

23. Luo T, Fu J, Xu A, Su B, Ren Y, Li N, Zhu J, Zhao X, Dai R, Cao J, et al. PSMD10/gankyrin induces autophagy to promote tumor progression through cytoplasmic interaction with ATG7 and nuclear transactivation of ATG7 expression. Autophagy. 2016;12(8):1355-71.

24. Lee YJ, Hah YJ, Kang YN, Kang KJ, Hwang JS, Chung WJ, Cho KB, Park KS, Kim ES, Seo HY, et al. The autophagy-related marker LC3 can predict prognosis in human hepatocellular carcinoma. PLoS One. 2013;8(11):e81540.

25. Zhang Y, Chen J, Wu SS, Lv MJ, Yu YS, Tang ZH, Chen XH, Zang GQ. HOXA10 knockdown inhibits proliferation, induces cell cycle arrest and apoptosis in hepatocellular carcinoma cells through HDAC1. Cancer Manag Res. 2019;11:7065-76.

26. Dong S, Ma X, Wang Z, Han B, Zou H, Wu Z, Zang Y, Zhuang L. YY1 promotes HDAC1 expression and decreases sensitivity of hepatocellular carcinoma cells to HDAC inhibitor. Oncotarget. 2017;8(25):40583-93.

27. Sun TY, Xie HJ, Li Z, Kong LF, Gou XN, Li DJ, Shi YJ, Ding YZ. miR-34a regulates $\mathrm{HDAC1}$ expression to affect the proliferation and apoptosis of hepatocellular carcinoma. Am J Transl Res. 2017;9(1):103-14.

28. Cao LL, Song X, Pei L, Liu L, Wang H, Jia M. Histone deacetylase HDAC1 expression correlates with the progression and prognosis of lung cancer: a meta-analysis. Medicine. 2017:96(31):e7663.

29. Cao LL, Yue Z, Liu L, Pei L, Yin Y, Qin L, Zhao J, Liu H, Wang H, Jia M. The expression of histone deacetylase HDAC1 correlates with the progression and prognosis of gastrointestinal malignancy. Oncotarget. 2017;8(24):3924153.

30. Lu ZH, Shvartsman MB, Lee AY, Shao JM, Murray MM, Kladney RD, Fan D, Krajewski S, Chiang GG, Mills GB, et al. Mammalian target of rapamycin activator RHEB is frequently overexpressed in human carcinomas and is critical and sufficient for skin epithelial carcinogenesis. Cancer Res. 2010; 70(8):3287-98

31. Liu F, Pan Z, Zhang J, Ni J, Wang C, Wang Z, Gu F, Dong W, Zhou W, Liu H. Overexpression of RHEB is associated with metastasis and poor prognosis in hepatocellular carcinoma. Oncol Lett. 2018;15(3):3838-45.

32. Huang $X Y$, Huang $Z L$, Zhang $P B$, Huang $X Y$, Huang J, Wang $H C$, Xu B, Zhou J, Tang ZY. CircRNA-100338 is associated with mTOR signaling pathway and poor prognosis in hepatocellular carcinoma. Front Oncol. 2019;9:392.

33. Liu X, Paila UD, Teraoka SN, Wright JA, Huang X, Quinlan AR, Gatti RA, Concannon P. Identification of ATIC as a novel target for Chemoradiosensitization. Int J Radiat Oncol Biol Phys. 2018;100(1):162-73.

34. Li M, Jin C, Xu M, Zhou L, Li D, Yin Y. Bifunctional enzyme ATIC promotes propagation of hepatocellular carcinoma by regulating AMPK-mTOR-S6 K1 signaling. Cell Communication Signaling. 2017;15(1):52

35. Duran A, Linares JF, Galvez AS, Wikenheiser K, Flores JM, Diaz-Meco MT Moscat J. The signaling adaptor p62 is an important NF-kappaB mediator in tumorigenesis. Cancer Cell. 2008;13(4):343-54.

36. Wei H, Guan JL. Blocking tumor growth by targeting autophagy and SQST M1 in vivo. Autophagy. 2015;11(5):854-5.

37. Saito T, Ichimura Y, Taguchi $K$, Suzuki T, Mizushima T, Takagi K, Hirose $Y$, Nagahashi M, Iso T, Fukutomi T, et al. p62/Sqstm1 promotes malignancy of HCV-positive hepatocellular carcinoma through Nrf2-dependent metabolic reprogramming. Nat Commun. 2016;7:12030.

38. Zhang Z, Li J, He T, Ouyang Y, Huang Y, Liu Q, Wang P, Ding J. The competitive endogenous RNA regulatory network reveals potential prognostic biomarkers for overall survival in hepatocellular carcinoma. Cancer Sci. 2019;110(9):2905-23.

39. Liu JL, Chen FF, Lung J, Lo CH, Lee FH, Lu YC, Hung CH. Prognostic significance of p62/SQSTM1 subcellular localization and LC3B in oral squamous cell carcinoma. Br J Cancer. 2014;111(5):944-54.

40. Schlafli AM, Adams O, Galvan JA, Gugger M, Savic S, Bubendorf L, Schmid RA, Becker KF, Tschan MP, Langer $R$, et al. Prognostic value of the autophagy markers LC3 and p62/SQSTM1 in early-stage non-small cell lung cancer. Oncotarget. 2016;7(26):39544-55.

41. Yang Q, Zhang MX, Zou X, Liu YP, You R, Yu T, Jiang R, Zhang YN, Cao JY, Hong $\mathrm{MH}$, et al. A prognostic bio-model based on SQSTM1 and N-stage identifies nasopharyngeal carcinoma patients at high risk of metastasis for additional induction chemotherapy. Clin Cancer Res. 2018;24(3):648-58.

42. Sasaki T, Lian S, Khan A, Llop JR, Samuelson AV, Chen W, Klionsky DJ, Kishi S. Autolysosome biogenesis and developmental senescence are regulated by both Spns1 and v-ATPase. Autophagy. 2017;13(2):386-403.

43. Yanagisawa H, Ishii T, Endo K, Kawakami E, Nagao K, Miyashita T, Akiyama K, Watabe K, Komatsu M, Yamamoto D, et al. L-leucine and SPNS1 coordinately ameliorate dysfunction of autophagy in mouse and human Niemann-pick type C disease. Sci Rep. 2017;7(1):15944.

44. Lyu L, Jin X, Li Z, Liu S, Li Y, Su R, Su H. TBBPA regulates calcium-mediated lysosomal exocytosis and thereby promotes invasion and migration in hepatocellular carcinoma. Ecotoxicol Environ Saf. 2020;192:110255.

\section{Publisher's Note}

Springer Nature remains neutral with regard to jurisdictional claims in published maps and institutional affiliations. 